\title{
Die Typologie der kaiserzeitlichen Prägungen aus Syedra
}

Die Gliederung des vorliegenden Typenkatalogs erfolgt primär nach Prägeherren (Kaiser und Mitglieder des Kaiserhauses); die einzige mir bekannt gewordene anonyme Prägung steht am Anfang des Katalogs.

Ausschlaggebend sind die Reversdarstellungen in signifikanten Kurzbeschreibungen; jedoch ist zu beachten, daß Gleichsetzungen mit mythologischen Figuren oftmals eine subjektive Interpretation darstellen. Reverslegenden werden nur dann angeführt, wenn sie nicht dem Ethnikon ( $\Sigma \mathrm{YE} \Delta \mathrm{PE} \Omega \mathrm{N})$ bzw. einer auf den Kleinnominalien vorkommenden Kurzform entsprechen. Auftretende Aversbüsten werden pauschal für den jeweiligen Prägeherren aufgelistet, da tiefergehende Untersuchungen der Avers-Revers-Kombinate eine breitere Materialbasis benötigen.

Als Wertangaben werden nur die unter Valerianus, Gallienus und Salonina explizit genannten (IA; H; $\varsigma ; \Gamma$ ) angeführt, doch kann dem Material mit aller gebotenen Vorsicht eine Dreigliederung an Wertstufen entnommen werden. Hierbei wurden als Kleinbronzen (KB) Stücke von 2 bis 5 Gramm, als Mittelbronzen Münzen von 7 bis 12 Gramm (MB) und Großbronzen (GB) jene mit mehr als 13 Gramm bezeichnet; bei geringen publizierten Stückmengen wurden subsidiär auch die Durchmesser als Kriterien herangezogen. Diese Klassifizierung beruht jedoch auf einer zu kleinen Materialdichte, um als endgültig bezeichnet werden zu können. Erst Statistiken mit breiter Basis und auch größerem räumlichen Umfang werden definitiv Aufschluß über mögliche Nominalien geben können.

Die Ausgangsbasis stellen insgesamt ca. 450 Stück aus großen, im Rahmen der Sylloge Nummorum Graecorum veröffentlichten, Sammlungen, der Numismatischen Zentralkartei am Institut für Numismatik und Geldgeschichte der Universität Wien und der Sammlung des Kunsthistorischen Museums in Wien dar.

Aus numismatischer Sicht ist die Typenvielfalt für Marcus Aurelius und Lucius Verus sowie für Maximus Caesar auffallend, noch dazu, wo für dessen Vater Maximinus Thrax die Zahl an Reverstypen sehr gering ist. Auch sind die Anzahl der Typen und überlieferten Belegstücke für Valerianus, Gallienus und Salonina - also aus der Endphase der Provinzialprägung - bemerkenswert. Generell ist festzustellen, daß neben variantenreichen agonistischen Typen die Gottheiten Ares und Demeter die Typologie von Syedra bestimmen.

\section{ANONYM, FRÜHE KAISERZEIT}

1) Avers: Büste der Athena nach rechts; Revers: Nike mit Kranz und Palmzweig geht nach rechts $(\mathrm{KB})$

SNG Pfalz 1132. (Abb. 1)

\footnotetext{
* Doktorandenstipendiat der Österreichischen Akademie der Wissenschaften.
} 


\section{Tiberius}

Kopf mit Lorbeerkranz nach rechts oder links

1) Göttin steht links mit Granatapfel und Stab (KB) SNG Pfalz 1133-1140; RPC 3405. (Abb. 2)

2) Göttin steht frontal mit Schale und Stab (KB) SNG Pfalz 1141-1144; RPC -. (Abb. 3)

\section{TIBERIUS ?}

Kopf mit Lorbeerkranz nach links

3) Kopf der Demeter nach rechts; rechts Kornähre und Mohnkapsel (KB) SNG Pfalz 1145. (Abb. 4)

\section{Traianus}

Kopf mit Lorbeerkranz nach rechts

1) Demeter steht nach links mit Ähren und Fackel (MB) SNG Paris 630; SNG Pfalz 1151 f. (Abb. 5)

2) Demeter steht nach rechts mit Fackel und Ähren (MB) SNG Levante Suppl. 71; SNG Paris 631. (Abb. 6)

3) Demeter sitzt nach links mit Ähren und Fackel (MB) SNG Levante 408; SNG Levante Suppl. 70. (Abb. 7)

4) Göttin (Artemis?) steht nach links mit Granatapfel und Stab; Köcher über Schulter? (KB) SNG Levante 407; Ziegler 104f. (Abb. 8)

5) Göttin steht nach links mit Granatapfel und Zweig (KB) SNG Levante 406; Ziegler 106-108. (Abb. 9)

\section{HadRIANUS}

Kopf mit Lorbeerkranz nach rechts; gepanzerte Büste mit Lorbeerkranz nach rechts

1) Demeter steht nach links mit Fackel und Ähren (MB) SNG Aulock 5897; SNG Levante 410. (Abb. 10)

2) Demeter sitzt nach links mit Ähren und Fackel (MB) SNG Pfalz 1162-1164; Ziegler 109.

3) Göttin steht nach links mit Schale und Stab (KB) SNG Paris 632; Ziegler 110-111.

4) Göttin steht nach links mit Granatapfel und Zweig (KB) SNG Pfalz 1159-1161.

5) Zeus steht nach links mit Zweig und Zepter; links von ihm Adler (GB) SNG Leypold 2631; SNG Paris 633. (Abb. 11)

\section{Antoninus Pius}

Kopf mit Lorbeerkranz nach rechts; Büste mit Paludamentum nach rechts

1) Demeter steht nach rechts mit Fackel und Ähren (MB) SNG Levante Supp 74.

2) Göttin steht nach links mit Schale und Stab (KB) SNG Paris 634. 
3) Zeus sitzt nach rechts mit Zepter und Schild (GB)

SNG Pfalz 1165. (Abb. 12)

\section{Marcus Aurelius}

Kopf mit Lorbeerkranz nach rechts oder links; Büste mit Paludamentum (über Küraß) und Lorbeerkranz nach rechts.

1) Artemis steht nach rechts mit Bogen und Granatapfel, über Schulter Köcher $(\mathrm{KB})$

Ziegler 115f; 118-120. (Abb. 13)

2) Athena Nikephoros steht nach links mit Speer, daran Schild gelehnt (KB) SNG Levante Supp. 76; SNG Paris 636; Ziegler 117. (Abb. 14)

3) Athena steht nach links zu Vase am Boden opfernd; Palmzweig in der Rechten (MB)

SNG Paris 635. (Abb. 15)

4) Athena steht nach links mit Speer und Schild (MB)

SNG Levante Supp. 77. (Abb. 16)

5) Tyche steht nach links mit Steuerruder und Füllhorn (KB)

SNG Pfalz 1178-1181; SNG Levante 411; Ziegler 113f. (Abb. 17)

6) Ares steht nach rechts mit Speer und Schild (KB)

SNG Levante 412; SNG Leypold 2634; SNG Pfalz 1176. (Abb. 18)

7) Dionysos steht nach links mit Kantharos und Thyrsos; links zu seinen FüBen Panther (MB)

SNG Leypold 2637. (Abb. 19)

8) Zeus steht nach links mit Schale und Zepter; links zu seinen Füßen Adler (GB)

SNG Levante Supp. 75. (Abb. 20)

9) Kaiser oder Lucius Verus mit Speer galoppiert auf Pferd über am Boden liegenden Parther (GB)

SNG Aulock 5899; SNG Levante 413; Münzen und Medaillen AG 41 (1970), 576. (Abb. 21)

10) Kaiser reitet nach rechts; rechter Arm zum Grußgestus erhoben (GB)

Peus 348 (2. 5. 1996), 461; Hirsch 175 (23. 9. 1992), 960. (Abb. 22)

\section{FAustina II}

drapierte Büste nach rechts

1) Aphrodite steht frontal, Kopf nach rechts, ihre Haare frisierend (MB) SNG Levante Supp. 78; SNG Paris 638; SNG Pfalz 1189-1192. (Abb. 23)

2) Demeter steht nach rechts mit zwei langen Fackeln (MB) SNG Pfalz $1193 \mathrm{f}$.

3) Tyche steht nach links mit Steuerruder und Füllhorn (KB) SNG Pfalz $1186 \mathrm{f}$.

4) Göttin steht nach rechts; Attribute unkenntlich (KB)

SNG Pfalz 1188. (Abb. 24) 


\section{LuCIUS VERUS}

Kopf mit Lorbeerkranz nach links; Büste mit Paludamentum (über Küraß) und Lorbeerkranz nach rechts

1) Artemis steht nach rechts mit Bogen und Granatapfel; über Schulter Köcher $(\mathrm{KB})$

SNG Levante 414; SNG Pfalz 1196-1199.

2) Athena steht nach links; Attribute unklar (MB) SNG Pfalz 1203.

3) Demeter steht nach links mit zwei langen Fackeln (MB) SNG Aulock 5900; SNG Paris 642. (Abb. 25)

4) Tyche steht nach links mit Zweig (?) und Füllhorn (KB) SNG Paris 643; SNG Pfalz 1200 f. (Abb. 26)

5) Dionysos steht nach links mit Kantharos und Thyrsos; links zu seinen FüBen Panther (MB)

SNG Levante Supp. 79; SNG Pfalz 1202.

6) Zeus steht nach links mit Schale und Zepter; links Schlange (GB) SNG Paris 639; SNG Pfalz 1205f. (Abb. 27)

7) Gottheit steht nach links mit Zweig und Stab (MB) Ziegler 122. (Abb. 28)

8) „Urteil über Ares“ Dike, Ares und Hermes stehen frontal; letzterer mit Geldbeutel und Kerykeion (GB) SNG Paris 641; Ziegler 121. (Abb. 29)

9) Lucius Verus reitet nach rechts; rechter Arm zum Grußgestus erhoben (GB) SNG Paris 640; SNG Pfalz $1207 \mathrm{f}$.

\section{LUCILLA}

drapierte Büste nach rechts

1) Aphrodite steht frontal, Kopf nach rechts, ihre Haare frisierend (MB) SNG Paris 645.

2) Demeter steht nach links mit Ähren und Fackel (MB) SNG Paris 644.

3) Demeter steht nach rechts mit zwei langen Fackeln (MB) SNG Levante 415; SNG Leypold 2642. (Abb. 30)

GETA

barhäuptiger Kopf nach rechts

1) Dionysos steht nach links mit Kantharos und Thrysos (KB) SNG Pfalz 1209. (Abb. 31)

\section{Severus Alexander}

Büste mit Paludamentum (über Küraß) und Lorbeerkranz nach rechts

1) Ares mit Speer, Parazonium und Chlamys steht nach rechts (GB) SNG Levante 417. (Abb. 32)

2) Zeus Nikephoros mit Zepter steht nach links; zu seinen Füßen Adler (GB) SNG Aulock 5901; SNG Levante 416. (Abb. 33)

3) Hygieia und Asklepios mit Stab stehen zueinander (GB) Hirsch 202 (25. 11. 1998), 521. (Abb. 34) 
4) Zwei Ringer kämpfend; darunter Palmzweig (GB)

Ziegler 123. (Abb. 35)

5) Preiskrone mit zwei Palmen (KB)

SNG Paris 647. (Abb. 36)

6) $\Sigma$ YE $\triangle \mathrm{PE} \Omega \mathrm{N}$ im Lorbeerkranz (GB)

SNG Paris 646. (Abb. 37)

\section{IULIA MAESA}

drapierte Büste nach rechts

1) Aphrodite steht frontal, Kopf nach rechts, ihr Haar frisierend (MB) SNG Levante 418; SNG Paris 648.

2) Athena steht nach links mit Speer und Schild (MB) SNG Leypold 2643; SNG Pfalz 1210.

3) Demeter steht nach links mit Stab und Ähren (MB)

Münchner Münzhandlung, Auktion 176 (18.-19. 3. 1980), 1387. (Abb. 38)

\section{IUlia Mamaea}

drapierte Büste nach rechts

1) Dionysos steht nach links mit Kantharos und Thyrsos; links zu seinen FüBen Panther (MB)

SNG Leypold 2644; Ziegler 124.

\section{Maximinus Thrax}

Büste mit Paludamentum (über Küraß) und Lorbeerkranz nach rechts

1) Ares steht nach rechts mit Schild und Speer (MB) SNG Aulock 5902; SNG Levante 419. (Abb. 39)

2) Hephaistos sitzt auf Felsen nach rechts mit Hammer und Schild (KB) SNG Leypold 2645. (Abb. 40)

3) Hermes steht nach links mit Geldbeutel und Kerykeion (MB) SNG Pfalz 1211. (Abb. 41)

4) $\Sigma \mathrm{YE} \triangle \mathrm{PE} \Omega \mathrm{N}$ im Lorbeerkranz (GB)

SNG Pfalz 1212.

\section{Maximus CAesar}

Büste mit Paludamentum (über Küraß) nach rechts; Büste mit Paludamentum (über Küraß) und Lorbeerkranz nach rechts

1) Aphrodite steht frontal, Kopf nach rechts, ihr Haar frisierend (MB) SNG Paris 653; SNG Pfalz 1213-1215; Ziegler 125.

2) Artemis steht nach rechts mit Bogen, Pfeil aus dem Köcher ziehend (KB) SNG Levante 422. (Abb. 42)

3) Athena steht nach links mit Schild und Speer (GB) SNG Paris 649; SNG Pfalz 1218. (Abb. 43)

4) Tyche steht nach links mit Steuerruder auf Globus und Füllhorn (MB) SNG Pfalz 1216. (Abb. 44)

5) Ares steht nach links mit Schild und Speer (MB) SNG Pfalz 1217; SNG Paris 652. 
6) Ares sitzt nach rechts mit Speer und Schild (GB)

SNG Paris 651. (Abb. 45)

7) Dionysos steht nach links mit Kantharos und Thrysos; links zu seinen FüBen Panther (KB) SNG Levante 424; Ziegler 126.

8) Zeus steht nach links mit Schale und Zepter (MB) Ziegler 127. (Abb. 46)

9) „Urteil über Ares“ Dike, Ares und Hermes stehen frontal; letzterer mit Geldbeutel und Kerykeion (GB) SNG Paris 650.

10) Göttin, Zeus und Demeter stehen nach links (GB) SNG Levante 421. (Abb. 47)

11) Preiskrone mit zwei Palmen (KB) SNG Levante 423.

12) $\Sigma Y E \Delta P E \Omega N$ im Lorbeerkranz (GB) SNG Levante 420.

\section{GoRDIANUs III}

Büste mit Paludamentum (über Küraß) und Lorbeerkranz nach rechts

1) Athena steht nach links mit Statuette (Athena?) und Speer (MB) SNG Pfalz 1222. (Abb. 48)

2) Ares steht nach rechts mit Speer und Schild; im Feld rechts Bogen (MB) SNG Levante 425. (Abb. 49)

3) Dionysos steht nach links mit Kantharos und Thrysos; links zu seinen FüBen Panther (MB)

SNG Pfalz 1221.

4) Zeus steht nach links mit Schale und Zepter (MB) SNG Levante 426.

5) „Urteil über Ares“ Dike, Ares und Hermes stehen frontal; letzterer mit Geldbeutel und Kerykeion (GB)

SNG Levante 427.

\section{Philippus Arabs}

Büste mit Paludamentum (über Küraß) und Lorbeerkranz nach rechts

1) Ares steht nach links mit Schild und Speer (GB) Auctiones 25 (19. 6. 1995), 598. (Abb. 50)

2) Hermes steht nach links mit Geldbeutel und Kerykeion (KB) SNG Pfalz 1223; Ziegler 129.

3) Zeus steht nach links mit Schale und Zepter (GB) Ziegler 128.

\section{Traianus Decius}

Büste mit Paludamentum (über Küraß) und Lorbeerkranz nach rechts

1) „Urteil über Ares“ Dike, Ares und Hermes stehen frontal; letzterer mit Geldbeutel und Kerykeion (GB)

SNG Paris 655; Ziegler $130 \mathrm{f}$. 
2) Gymnasiarchenbecken, darüber drei unterschiedlich große zylindrische Henkelgefäße; links und rechts je ein Palmzweig (Reverslegende $\Sigma$ YE$\triangle \mathrm{PE} \Omega \mathrm{N}$ ГYMNA $\Sigma$ IAPXIA) (GB)

SNG Paris 654; Ziegler 132. (Abb. 51)

3) Zwei zylindrische Gefäße und Gymnasiarchenbecken; darüber eine Palme (Reverslegende $\Sigma$ YE $\triangle$ PE $\Omega N$ TYMNA $\Sigma$ IAPXIA) (GB)

CNG 42 (29. 5. 1997), 1035. (Abb. 52)

\section{Etruscilla}

drapierte Büste mit Stephane nach rechts

1) „Urteil über Ares“ Dike, Ares und Hermes stehen frontal; letzterer mit Geldbeutel und Kerykeion (GB)

SNG Paris 656 .

2) Gymnasiarchenbecken, darüber drei unterschiedlich große zylindrische Henkelgefäße; links und rechts je ein Palmzweig (Reverslegende $\Sigma$ YE$\triangle \mathrm{PE} \Omega \mathrm{N}$ ГYMNA $\Sigma$ IAPXIA) (GB)

SNG Levante Supp. 82; Ziegler 133.

\section{Herennius Etruscus}

Büste mit Paludamentum nach rechts

1) Hades sitzt nach links mit Schale und Stab; zu seinen Füßen Kerberos; links von ihm Persephone (MB)

SNG Paris 657. (Abb. 53)

2) $\Sigma \mathrm{YE} \Delta \mathrm{PE} \Omega \mathrm{N}$ im Lorbeerkranz (MB)

SNG Levante 428. (Abb. 54)

\section{Trebonianus Gallus}

Büste mit Paludamentum (über Küraß) und Lorbeerkranz nach rechts

1) Hades mit Persephone in Quadriga nach rechts fahrend; diese gelenkt von Hermes; Fackel und Kantharos zu Füßen der Pferde (GB) SNG Levante 429; SNG Leypold 2647; Ziegler 134. (Abb. 55)

2) „Urteil über Ares“ Dike, Ares und Hermes stehen frontal; letzterer mit Geldbeutel und Kerykeion (GB)

SNG Aulock 5903.

3) Gymnasiarchenbecken, darüber drei unterschiedlich große zylindrische Henkelgefäße; links und rechts je ein Palmzweig (Reverslegende $\Sigma$ YE$\triangle \mathrm{PE} \Omega \mathrm{N}$ ГYMNA $\Sigma$ IAPXIA) (GB)

SNG Pfalz 1224.

\section{Volusianus}

Büste mit Paludamentum (über Küraß) und Lorbeerkranz nach rechts

1) Demeter steht nach rechts mit zwei langen Fackeln (MB) SNG Levante 430; SNG Pfalz 1226.

2) $\Sigma \mathrm{YE} \Delta \mathrm{PE} \Omega \mathrm{N}$ im Lorbeerkranz (MB)

SNG Levante 431; SNG Pfalz 1225. 


\section{VALERIANUS}

Büste mit Paludamentum (über Küraß) und Lorbeerkranz nach rechts

Mittelbronze mit Wertzeichen $\mathrm{H}$ im Avers

1) Ares steht nach rechts mit Speer und Schild SNG Paris 658; SNG Pfalz 1227; Ziegler 139f.

Großbronze mit Wertzeichen IA im Avers

2) „Urteil über Ares“ Dike, Ares und Hermes stehen frontal; letzterer mit Geldbeutel und Kerykeion

SNG Paris 662; Ziegler $137 \mathrm{f}$.

3) Zwei Ringer (Reverslegende $\Sigma \mathrm{YE} \Delta \mathrm{PE} \Omega \mathrm{N} \Theta \mathrm{EMI} \Sigma$ ) SNG Aulock 5904; SNG Paris 661; Ziegler 135.

4) Gymnasiarchenbecken, darüber drei unterschiedlich große zylindrische Henkelgefäße; links und rechts je ein Palmzweig (Reverslegende $\Sigma$ YE$\triangle$ PE $\Omega N$ TYMNA $\Sigma$ IAPXIA)

SNG Paris 659.

5) Zwei Preiskronen auf Tisch mit Palmen darin (Reverslegende $\Sigma$ YE$\triangle \mathrm{PE} \Omega \mathrm{N} \Theta \mathrm{EMI} \Sigma)$

SNG Pfalz 1228. (Abb. 56)

6) $\Sigma$ EMNH $\Sigma$ EN $\triangle \mathrm{O} \Xi \mathrm{OTEPA} \Sigma \Sigma \mathrm{YE} \Delta \mathrm{PE} \Omega \mathrm{N}$ im Lorbeerkranz SNG Paris 660; SNG Pfalz 1229; Ziegler 136.

\section{GaLlienus}

Büste mit Paludamentum (über Küraß) und Lorbeerkranz nach rechts

Kleinbronze mit Wertzeichen $\Gamma$

1) Demeter steht nach rechts mit zwei langen Fackeln Ziegler 154.

2) Tyche steht nach rechts mit Steuerrunder und Füllhorn SNG Levante 433; Ziegler 155.

Mittelbronze mit Wertzeichen $\varsigma$

3) Gymnasiarchenbecken, darüber drei unterschiedlich große zylindrische Henkelgefäße; links und rechts je ein Palmzweig (Reverslegende $\Sigma Y$ Y $\triangle \mathrm{PE} \Omega \mathrm{N} \Gamma \mathrm{YMNA} \Sigma \mathrm{IAPXIA)}$ SNG Pfalz 1231.

Mittelbronze mit Wertzeichen $\mathrm{H}$

4) „Urteil über Ares“ Dike, Ares und Hermes stehen frontal; letzterer mit Geldbeutel und Kerykeion SNG Levante 434; Ziegler 141.

5) Zwei Preiskronen auf Tisch mit Palmen darin (Reverslegende $\Sigma$ YE$\triangle \mathrm{PE} \Omega \mathrm{N} \Theta \mathrm{EMI} \Sigma)$

SNG Levante 435.

Großbronze mit Wertzeichen IA

1) Ares sitzt nach rechts mit Speer und Schild SNG Paris 666; Ziegler 148-152.

2) „Urteil über Ares“ Dike, Ares und Hermes stehen frontal; letzterer mit Geldbeutel und Kerykeion

SNG Paris 663f; Ziegler 145-147. 
3) Zwei Ringer (Reverslegende $\Sigma$ YE $\triangle \mathrm{PE} \Omega \mathrm{N} \Theta \mathrm{EMI} \Sigma$ )

SNG Aulock 5905; SNG Paris 665; Ziegler 142. (Abb. 57)

4) Gymnasiarchenbecken, darüber drei unterschiedlich große zylindrische Henkelgefäße; links und rechts je ein Palmzweig (Reverslegende $\Sigma$ YE$\triangle$ PEON $\Gamma Y M N A \Sigma I A P X I A)$ SNG Pfalz 1232-1236; Ziegler $143 \mathrm{f}$.

5) $\Sigma$ EMNH $\Sigma$ EN $\triangle$ OXOTEPA $\Sigma \Sigma$ YE $\triangle$ PEON im Lorbeerkranz SNG Pfalz 1247 f; SNG Paris 667.

\section{SALONINA}

drapierte Büste mit Stephane nach rechts

Großbronze mit Wertzeichen IA

6) Demeter steht nach links mit Zweig und Fackel SNG Paris 673; SNG Pfalz 1263.

7) Demeter steht nach rechts mit zwei langen Fackeln SNG Paris 671; Ziegler 163-167.

8) Demeter steht nach rechts mit zwei kurzen Fackeln Lindgren 911; SNG Paris 670; Ziegler 162. (Abb. 58)

9) Ares steht nach rechts mit Speer und Schild SNG Aulock 5906; SNG Paris 669; Ziegler 168.

10) Ares sitzt nach rechts mit Speer und Schild SNG Paris 668; Ziegler $160 \mathrm{f}$.

11) Zwei Ringer (Reverslegende $\Sigma$ YE $\Delta$ PE $\Omega N$ NEMI $\Sigma$ ) SNG Pfalz 1256-1260; Ziegler $158 \mathrm{f}$.

12) Gymnasiarchenbecken, darüber drei unterschiedlich große zylindrische Henkelgefäße; links und rechts je ein Palmzweig (Reverslegende $\Sigma$ YE$\triangle$ PE $\Omega N$ ГYMNA $\Sigma$ IAPXIA)

SNG Paris 672; SNG Pfalz $1261 \mathrm{f}$.

13) Zwei Preiskronen auf Tisch mit Palmen darin (Reverslegende $\Sigma Y E$ $\triangle \mathrm{PE} \Omega \mathrm{N} \Theta \mathrm{EMI} \Sigma)$ SNG Paris 674.

14) $\Sigma$ EMNH $\Sigma$ EN $\Delta \mathrm{O} \Xi \mathrm{OTEPA} \Sigma \Sigma \mathrm{YE} \Delta \mathrm{PE} \Omega \mathrm{N}$ im Lorbeerkranz SNG Paris 675 f; Ziegler 156 f. (Abb. 59)

\section{ABKÜRZUNGEN}

$\mathrm{RPC}$

SNG Aulock

SNG Levante

SNG Levante Supp. $=$ Sylloge Nummorum Graecorum Switzerland 1. Levante - Cilicia. Supplement 1 (bearbeitet von E. LeVANTE), Zürich 1993.

SNG Leypold = Sylloge Nummorum Graecorum Österreich. Sammlung Leypold, Kleinasiatische Münzen der Kaiserzeit, Band 2, Phrygien - Kommagene (bearbeitet von W. SZAIVERT und C. DABURON) (= Veröf- 
fentlichungen des Instituts für Numismatik und Geldgeschichte 11), im Druck.

Lindgren

= H. LindGren, Ancient Greek Bronze Coins from The Lindgren Collection, Band III, Berkeley 1993.

SNG Paris

= Sylloge Nummorum Graecorum France 2. Cabinet des Médailles, Cilicie (bearbeitet von E. Levante), Paris 1993.

SNG Pfalz

$=$ Sylloge Nummorum Graecorum Deutschland. Pfälzer Privatsammlungen Band 6, Isaurien und Kilikien (bearbeitet von R. ZIEGLER), München 2001.

Ziegler

$=$ R. ZIEGLER, Münzen Kilikiens aus kleineren deutschen Sammlungen (= Vestigia 42), München 1988.

ABBILDUNGEN**

Reverstypen, die unter mehreren Prägeherren auftreten, sind nur einmal abgebildet

1) SNG Pfalz 1132

2) SNG Levante 405

3) SNG Pfalz 1141

4) SNG Pfalz 1145

5) SNG Paris 630

6) SNG Levante Supplement 71

7) SNG Levante Supplement 70

8) SNG Pfalz 1146

9) Ziegler 107

10) SNG Levante 410

11) SNG Paris 633

12) SNG Pfalz 1165

13) SNG Pfalz 1169

14) SNG Levante Supplement 76

15) SNG Paris 635

16) SNG Levante Supplement 77

17) SNG Pfalz 1178

18) SNG Pfalz 1176

19) SNG Leypold 2637

20) SNG Levante Supplement 75

21) GM 108 (3. 4. 2001), 1535

22) Peus 348 (2. 5. 1996), 461

23) SNG Pfalz 1189

24) SNG Pfalz 1188

25) SNG Paris 642

26) SNG Pfalz 1200

27) SNG Paris 639

28) Ziegler 122

29) Ziegler 121

30) SNG Levante 415
31) SNG Pfalz 1209

32) SNG Levante 417

33) SNG Levante 416

34) Hirsch 202 (25. 11. 1998), 521.

35) Ziegler 123

36) SNG Paris 647

37) SNG Paris 646

38) Münchner Münzhandlung, Auktion 176 (18.-19. 3. 1980), 1387

39) SNG Levante 419

40) SNG Leypold 2645

41) SNG Pfalz 1211

42) SNG Levante 422

43) SNG Paris 649

44) SNG Pfalz 1216

45) SNG Paris 651

46) Ziegler 127

47) SNG Levante 421

48) SNG Pfalz 1222

49) SNG Levante 425

50) Auctiones 25 (19. 6. 1995), 598.

51) Ziegler 132

52) CNG 42 (29. 5. 1997), 1035.

53) SNG Paris 657

54) SNG Levante 428

55) SNG Levante 429

56) SNG Pfalz 1228

57) SNG Paris 665

58) Ziegler 162

59) Aufhäuser 5 (5. 10. 1988), 453

** Mein Dank gilt Univ.-Prof. Dr. W. Szaivert für seine Hilfe und die Erlaubnis, Abbildungen aus der SNG Leypold zu verwenden. 


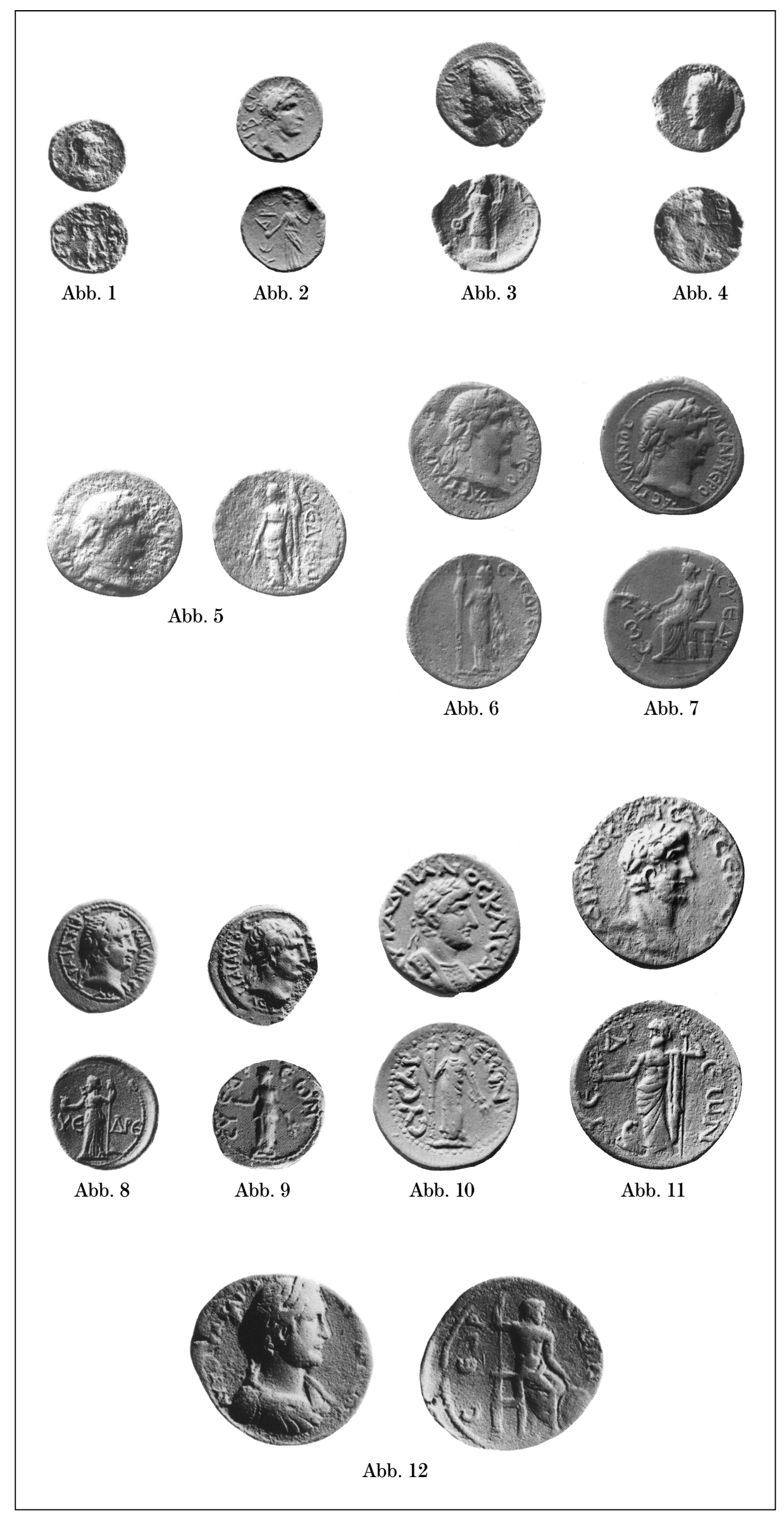




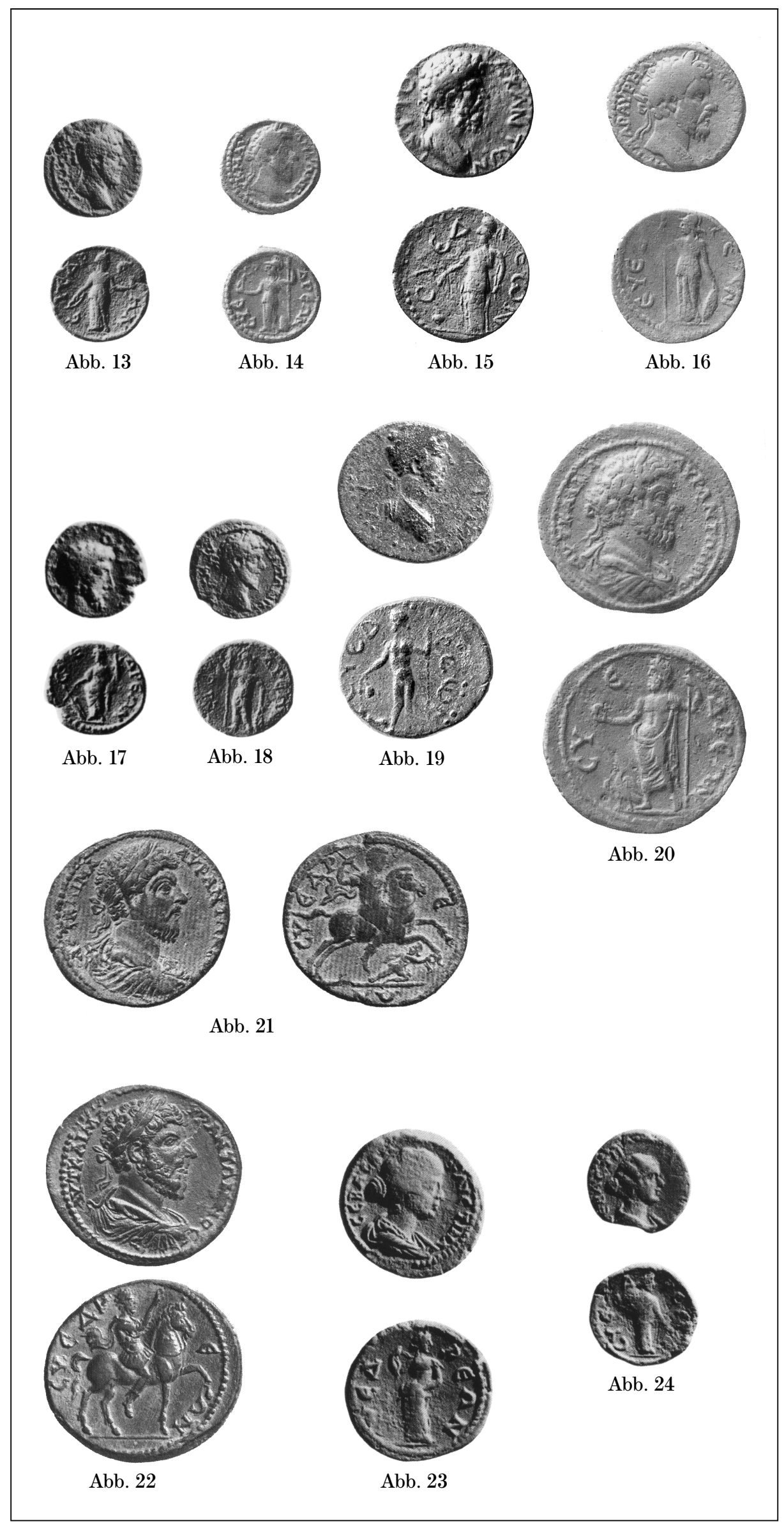




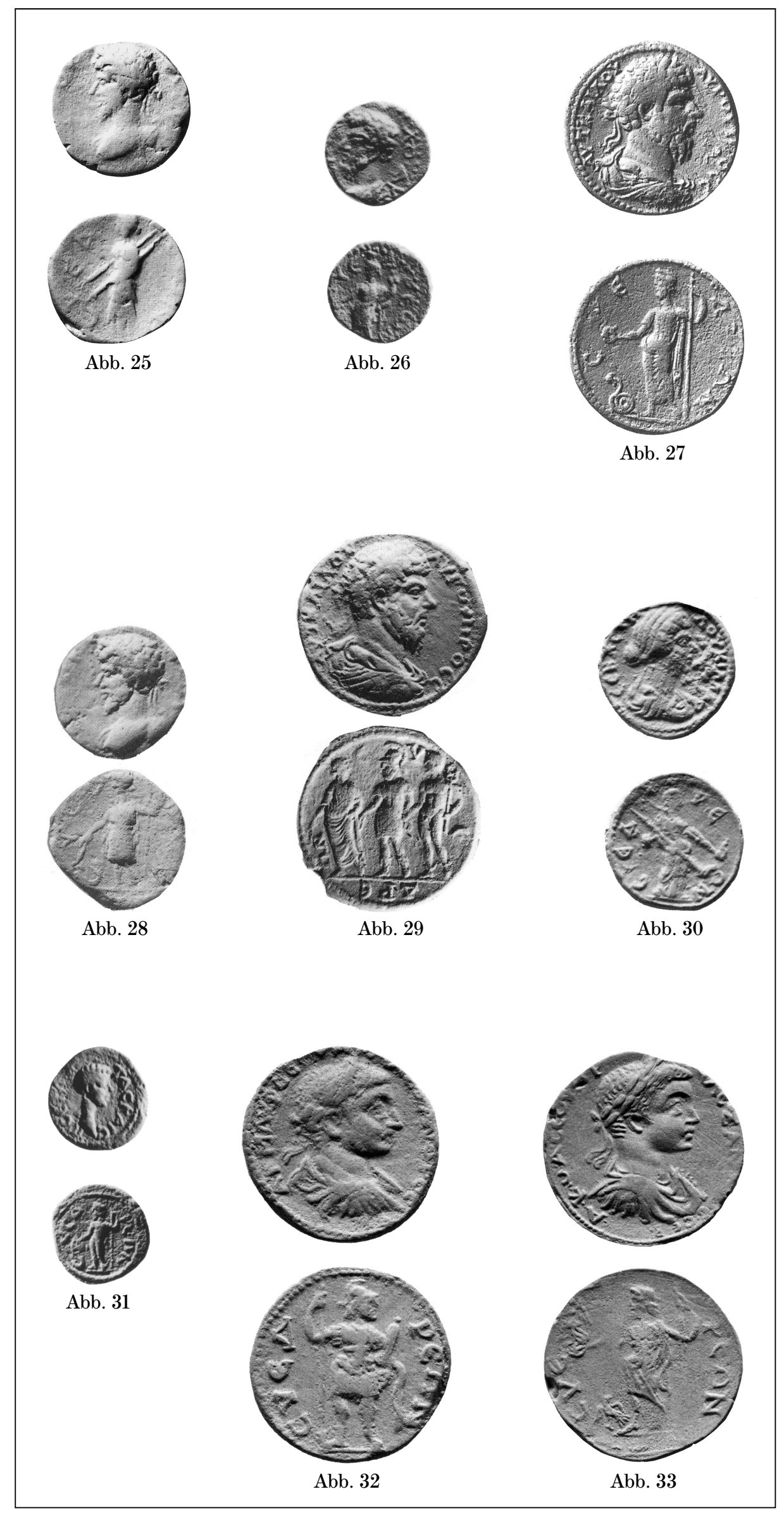




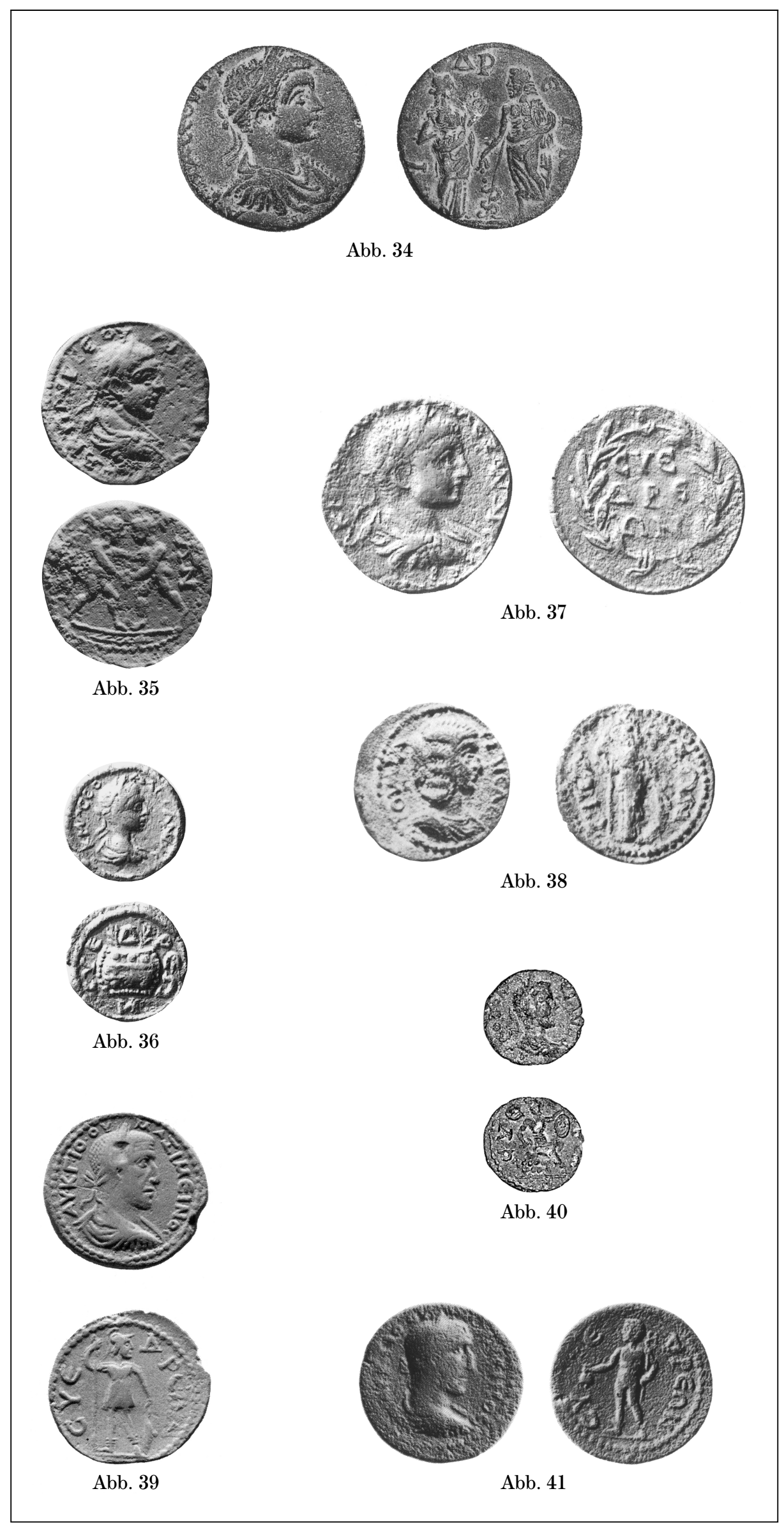




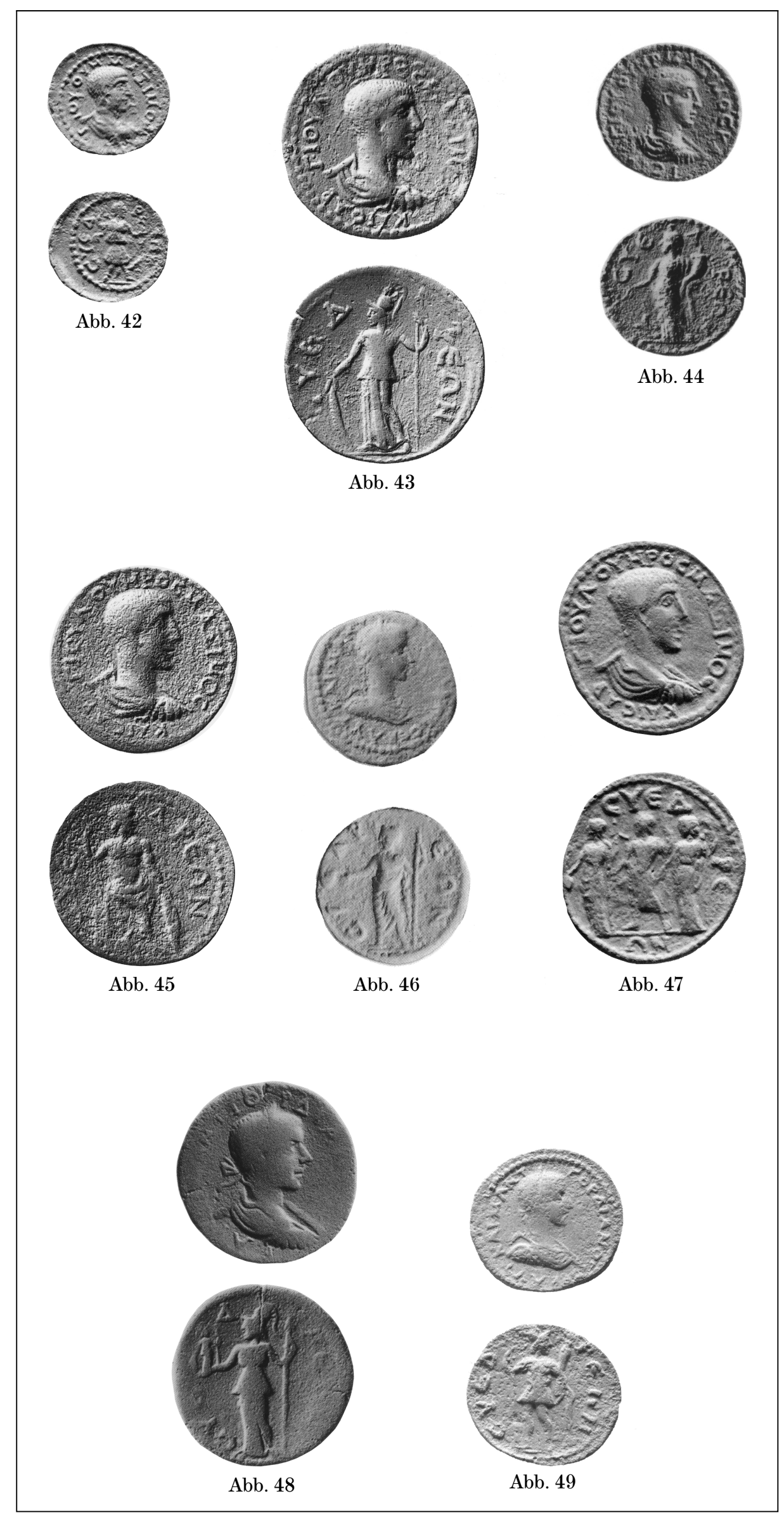




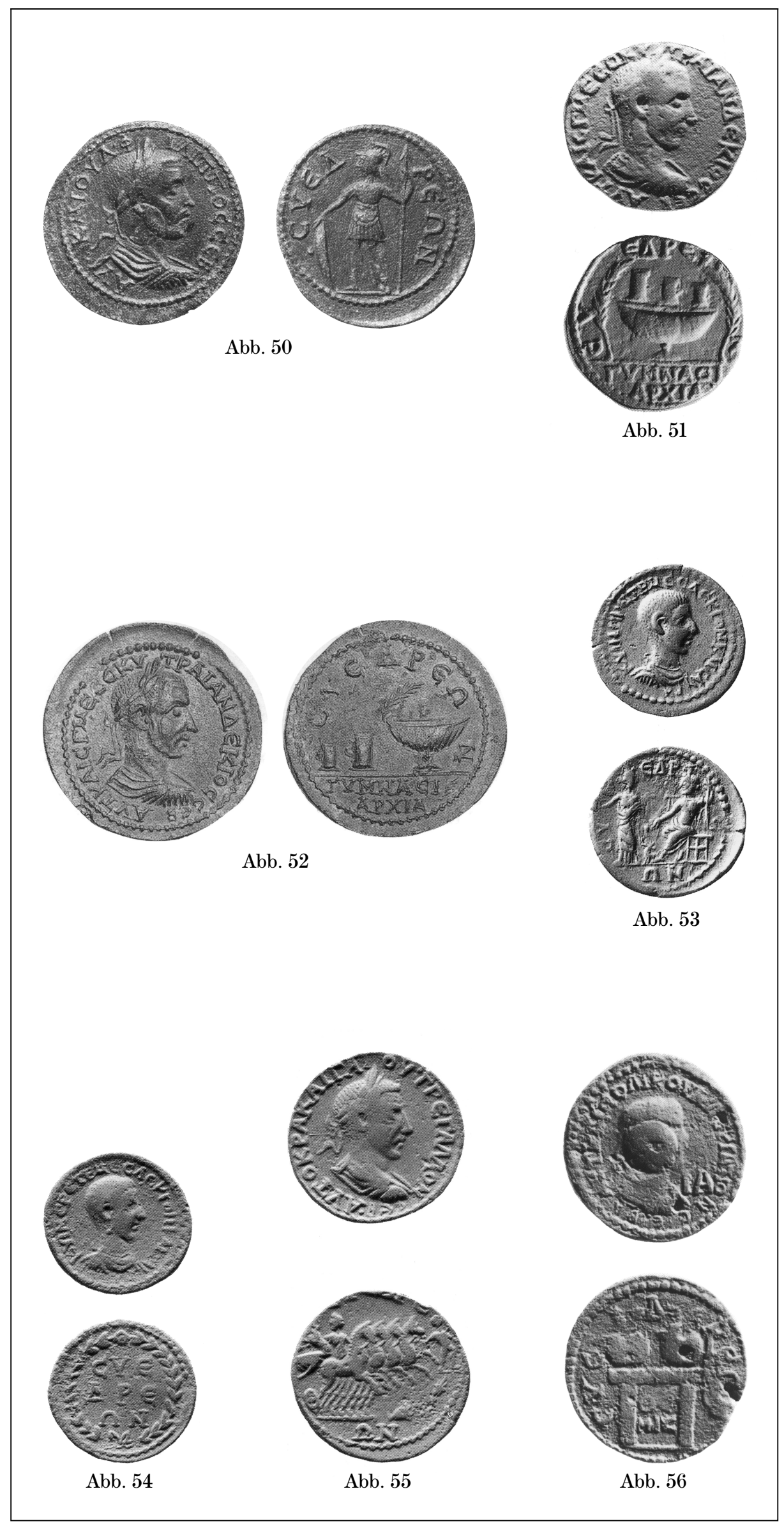




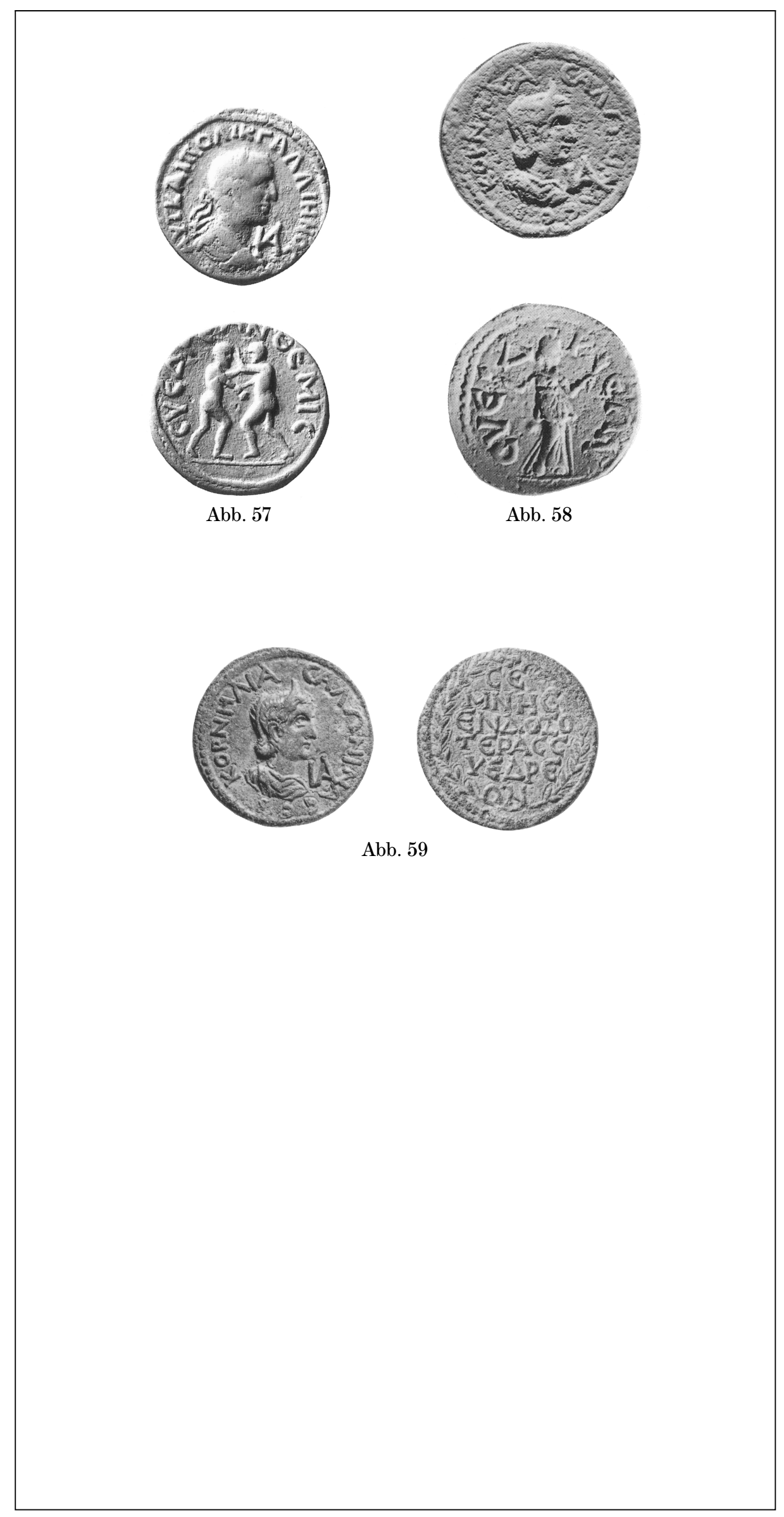

